\title{
PROFESSIONAL DIRECTORY
}

\section{SPACE RESERVED FOR CERTIFIED CONSULTING METEOROLOGISTS}

A.H. GLENN AND ASSOCIATES

ALFRED H. GLENN

\section{Certified Consulting Meteorologist}

Consultants in Meteorology and Oceanography since 1946• Professional Engineer

New Orleans Lakefront Airport

$504-241-2222$

P. O. Box 26337

New Orleans, LA 70126

FAX 504-241-2224

Telex 58-7445

Cable: GLENN, New Orleans

\section{WEATHER SERVICES CORPORATION}

JOHNE. WALLACE PETER R. LEAVITT JOHNP. MURPHY

\section{Certified Consulting Meteorologists}

Worldwide Forecasts, Climatological Surveys \& Data Banks for: Business $\bullet$ industry $\bullet$ Agribusiness $\bullet$ Marine $\bullet$ Media

Telex 92-3330 Bedford: $617-275-8860$

131A Great Rd

Bedford, MA 01730

New York: 212-267-1500 Houston: 713-738-1495 FAX 617-271-0178

NORTH AMERICAN WEATHER

\section{CONSULTANTS}

A TRC COMPANY

KEITH J. BROWN, PRESIDENT

\section{Certified Consulting Meteorologist}

Weather Modification • Air Quality Surveys \& Field Studies • Applied Research • Forensic Meteorology

1293 West 2200 South

Salt Lake City, UT 84119-1471

$801-972-3500$ FAX 801-972-3813

AEROMET, INC.

D. RAY BOOKER, PH.D., PRESIDENT

Certified Consulting Meteorologist

Instrumented Aircraft $\bullet$ Aerospace Meteorology $\bullet R \& D \bullet$ Forecasts

P.O. Box 701767

Tulsa, OK 74170-1767

918-299-2621

LOREN W. CROW CONSULTANTS, INC. LOREN W. CROW, PRESIDENT

\section{Certified Consulting Meteorologist}

Consulting \& Applied Meteorological Services

\section{MURRAY AND TRETTEL,} INCORPORATED

JOHN R. MURRAY

JOHNP. BRADLEY

DENNIS W. TRETTEL

\section{Certified Consulting Meteorologists}

Founded 1946, Micro/Operational Forecasting •Media • Air Quality/ Meteorological/PSD Monitoring/Studies $\bullet$ Forensic Research $\bullet$ Dispersion Modeling $\bullet$ Nuclear Emergency Support

414 W. Frontage Rd. (708)446-7800/FAX (708)446-1420 Northfield, IL 60093 (312) $273-5600$

WEATHER CORPORATION OF AMERICA

ARMAND R. IACCHEO EUGENE G. GUEBER

\section{Certified Consulting Meteorologists}

Operational Forecasts/Applied Research

514 Earth City Expressway, Ste. 321

314-344-8855

Cable: Weather

St. Louis, MO 63045 FAX 314-344-8866

\section{CLIMATOLOGICAL CONSULTING} CORPORATION

WALTER A. BOHAN

\section{Certified Consulting Meteorologist}

Specializing in Forensic Meteorology
P.O. Box 736

Park Ridge, IL 60068

708-825-3677
150 Shope Creek Road Asheville, NC 28805 704-298-4237

\section{METRO MONITORING SERVICES}

ERWIN K. KAUPER

\section{Certified Consulting Meteorologist}

Environmental Impact \& Site Surveys • Air Pollution Field Studies • Diffusion Analysis • Weather \& Air Quality Forecasting • Air Pollution Monitoring Instrumentation

P.O. Box 4584

Covina, CA 91723

$818-332-8411$

\section{RICHARD E. CALE}

\section{Certified Consulting Meteorologist}

Environmental Research Applications • Aviation Weather Investigations • Planning Services

Air Force Village West

21330 Twining Ave.

Riverside, CA 92518 


\section{SPACE RESERVED FOR CERTIFIED CONSULTING METEOROLOGISTS}

WEATHER RESEARCH CENTER

JOHN C. FREEMAN, DIRECTOR OF RESEARCH

JILLF. HASLING, DIRECTOR OF OPERATIONS

\section{Certified Consulting Meteorologists}

Worldwide Weather \& Oceanographic Forecasting • Climatology • Expert Testimony • Research in Meteorology \& Oceanography • Wave Spectra $\bullet$ Software Development $\bullet$ Observing Network Design

3227 Audley

Houston, TX 77098

$713-529-3076$

AEROVIRONMENT INC

PAUL B. MACCREADY, JR., PH.D.

KENNETH H. UNDERWOOD, PH.D.

WILLIAM C. BRICK

\section{Certified Consulting Meteorologists}

Air Quality/Meteorology/Hazardous Waste Monitoring • Diffusion Modeling • Tracer Experiments • Instrumentation-Acoustic Radar, Air Samplers • Wind Energy Assessments/Wind Engineering

P.O. Box 5031

Monrovia, CA 91017-7131 Santa Barbara, CA 805-967-7699 818-357-9983 Denver, CO 303-989-2667

McVEHIL-MONNETT ASSOCIATES, INC. GEORGE E. McVEHIL, PH.D.

\section{Certified Consulting Meteorologist}

Industrial Meteorology • Air Quality Modeling • Permitting • Meteorological Analysis and Consulting Services - Air Toxics/Hazardous Waste Monitoring and Analysis • Expert Testimony

Fairway at Inverness

44 Inverness Drive East

Building C, Suite B

303-790-1332

Englewood, CO 80112 FAX 303-790-7820

SIMPSON WEATHER ASSOCIATES, INC. M. GARSTANG, PH.D. R.H. SIMPSON, PH.D.

M. GARSTANG, PH.D.

\section{Cerified Consulting Meteorologists}

Environmental Hazard/Risk Assessment • Air Quality ModelingMonitoring $\bullet$ Instrumentation - towers, lidars, surface network $\bullet$ Software Development • Environmental Simulations

809 E. Jefferson St

804-979-3571

Charlottesville, VA 22902

FAX: 804-979-5599

\section{CLIMATOLOGICAL CONSULTING CORPORATION}

WILLIAM H. HAGGARD

DR. WERNER A. BAUM

STEPHEN W. SMUTZ

\section{Certified Consulting Meteorologists}

Specializing in Forensic Meteorology

150 Shope Creek Road

Asheville, NC 28805-9795

$704-298-4237$

\section{W. BOYNTON BECKWITH}

\section{Certified Consulting Meteorologist}

Aviation-Accident Investigation \& Operational Applications Mesometeorological Analyses • Climatological Studies • Expert Testimony

14728 Caminito Orense Oeste

San Diego, CA 92129

$619-672-1565$

TRC ENVIRONMENTAL CORP.

GALEF. HOFFNAGLE

ROBERT J.BALL

DOUGLASR. MURRAY

LYSA G. MODICA

\section{Certified Consulting Meteorologists}

Environmental Consulting \& Research • Applied Meteorology • Air Quality and Meteorological Monitoring $\bullet$ Diffusion Modeling $\bullet$ Tracer Studies • Air Toxics Monitoring

5 Waterside Crossing

Windsor, CT 0609

Denver, CO 303-792-5555

203-289-8631

Los Angeles, CA 714-581-6860 Seattle, WA 206-778-5003

APPLIED METEOROLOGY, INC.

JOHN W. HATHORN

Certified Consulting Meteorologist

Meteorological Consulting \& Analysis • Air Quality Modeling \& Monitoring $\bullet$ Site Selection \& Permitting $\bullet$ Environmental Data Acquisition Systems \& Network with Remote-Control

9000 Southwest Freeway

Suite 400

Houston, TX 77074

713-777-0106

INTERNATIONAL CENTER FOR THE SOLUTION OF ENVIRONMENTAL PROBLEMS

JOSEPHL. GOLDMAN, PH.D., TECHNICAL DIRECTOR

Certified Consulting Meteorologist

Broad Scope Assessments by Interdisciplinary Experts Worldwide -Specialize in Mesometeorological Variations • Climate Change • Problems Posed, Solutions Implemented \& Demonstrated

535 Lovett Blvd.

Houston, TX 77006

713-527-8711 Telex 775-167

OCEANROUTES, INC ROBERT L.COHEN

JAY P. GANZ

\section{Certified Consulting Meteorologists}

Consultants in Meteorology \& Oceanography • Optimum Ship Rout ing • Site Specific Spectral Wave Forecasting • Specialized Graphics 680 W. Maude Ave.

Sunnyvale, CA 94086-3518

408-245-3600 FAX 408-245-5301 Telex 184-802 Cable OCEANROUTES Aberdeen, Hong Kong, London, New York, Perth, Shanghai, Melbourne, San Francisco, Seoul, Singapore, and Tokyo

Certified Consulting Meteorologist: The certification program of the American Meteorological Society is aimed at fostering the establishment and maintenance of a high level of professional competency, and mature and ethical counsel, in the field of consulting meteorology. Requirements of knowledge, experience, and character are determined by a five-person board. Objectives of the program and application procedures are described in full detail in the August 1993BULLETIN (pp. 1550-1554). 


\section{PROFESSIONAL DIRECTORY}

\section{SPACE RESERVED FOR CERTIFIED CONSULTING METEOROLOGISTS}

\section{DAMES \& MOORE \\ PERRY W. FISHER, PH.D}

A. ROGER GREENWAY

\section{Certified Consulting Meteorologists}

Industrial Meteorology • Air Quality Modeling/Monitoring • PSD Permit Application Preparation • Expert Testimony • Climatological Studies $\bullet$ Nuclear Meteorological Services and Analyses

Rolling Meadows, IL

Cranford, $\mathrm{N} J$ 708-228-0707 908-272-8300

\section{AEROCOMP, INC.}

JOSEPH A. CATALANO

\section{Certified Consulting Meteorologist}

Air Quality Monitoring \& Analysis • Atmospheric Modeling • Climatological Studies • Data Management Systems \& Software

3303 Harbor Blvd.

Costa Mesa, CA 92626

AEROMATRIX INC.

CONRAD J. MASON, PH.D., PRESIDENT

\section{Certified Consulting Meteorologist}

Solar Energy Surveys - Biometeorology - Meteorological \& Air Quality Monitoring $\bullet$ Dispersion Modeling $\bullet$ Site Surveys $\bullet$ Expert Testimony

3640 E. Huron River Dr.

Ann Arbor, Ml 48104

313-971-2244

\section{ATMOSPHERICS INCORPORATED}

THOMAS J. HENDERSON, PRESIDENT

\section{Certified Consulting Meteorologist}

Weather Modification Operation - Cloud Physics Research • Airborne Instrumentation •Weather Radar Applications • Aerosol Measurements • General Meteorological Services

5652 E. Dayton Ave.

Fresno, CA 93727

EDWIN X BERRY \& ASSOCIATES

EDWIN X BERRY, PH.D.

\section{Certified Consulting Meteorologist}

Atmospheric Physics $\bullet$ Climatological Studies $\bullet$ Simulation Models • Specialized Forecasting $\bullet$ Expert Testimony
6040 Verner Avenue

Sacramento, CA 95841-2032
$916-344-7222$

FAX 916-344-7243
209-291-5575 FAX 209-291-5579
DAMES \& MOORE

STANLEY J. KRIVO GEORGEC.HOWROYD, PH.D.

JAMES W. LITTLE ROBERT G. ALLEN

\section{Certified Consulting Meteorologists}

Industrial Meteorology - Air Quality Modeling/Monitoring • PSD Permit Application Preparation • Expert Testimony $\bullet$ Climatological Studies • Nuclear Meteorological Services and Analyses

Atlanta, GA

404-262-2915

\section{WEATHER CONSULTANTS} INCORPORATED

JOHN T. WALSER, PRESIDENT

\section{Certified Consulting Meteorologist}

Specializing in Weather Modification Design \& Operations $\bullet$ Weathe \& Climate Information Service and Expert Testimony $\bullet$ Data Acquisition \& Analysis • Wind Energy Assessment \& Siting

P.O. Box 3414

707-829-5370

Santa Rosa, CA 95402

Telex 38-0795

\section{LYLE E. BROSCHE}

\section{Certified Consulting Meteorologist}

Specializing in Long-Range Weather Outlooks and Consulting

1347 Silver Lake Drive

Melbourne, FL 32940

AWD TECHNOLOGIES

AMIRAM ROFFMAN, PH.D.

\section{Certified Consulting Meteorologist}

Air Quality Consulting, Diffusion Modeling, Air Toxics $\bullet$ Monitoring \& Field Programs • Environmental Studies, Hazardous \& Solid Waste Programs $\bullet$ Risk Assessment $\bullet$ Health \& Safety $\bullet$ Remediation

Penn Center West, Bldg. III, Suite 400

Pittsburgh, PA 15276

412-788-2717

Regional offices in Rockville, MD; Fairfield, NJ; Tampa, FL; Houston, TX; Salt Lake City, UT; San Franscico, CA; Los Angeles, CA

WILKENS WEATHER TECHNOLOGIES DIVISION (an Air Routing International Corporation) RICHARD B. WILKENS, EXECUTIVE VICE-PRESIDENT

\section{Certified Consulting Meteorologist}

Specialists in Marine, Aviation \& Industrial Forecasting Worldwide • Hindcast \& Climatological Studies • Computerized Flight Plans 


\section{SPACE RESERVED FOR CERTIFIED CONSULTING METEOROLOGISTS}

FREESE-NOTIS WEATHER, INC.

HARVEY FREESE, M.S. CHARLES NOTIS. M.S

\section{Certified Consulting Meteorologist}

Worldwide forecasts for: Commodities \& Agriculture, Media, State \& Municipal Government, Utilities, Construction, Climatological Research, Forensic Meteorology \& Expert Testimony

2411 Grand Ave.

Des Moines, IA 50312

$515-282-9310$ or $515-282-9243$ FAX 515-282-6832

ENGINEERING-SCIENCE, INC.

DAVID B. SMITH

\section{Certified Consulting Meteorologists}

Air Toxics/Air Quality/MeteorologicalMonitoring •Dispersion Modeling $\bullet$ Source Testing $\bullet$ Risk Assessment $\bullet$ Applied Climatology $\bullet$ Clean Air Act Consulting and Permitting

617-859-2000

Prudential Center

Boston, MA 02199

Regional Offices in

California, Louisiana,

Texas, and Virginia

\section{ATEK CORPORATION}

GEOFFREY E. HILL, PH.D., PRESIDENT

\section{Certified Consulting Meteorologist}

Atmospheric Technology \& Engineering - Weather Modification Research \& Operations $\bullet$ Remote Sensing $\bullet$ Computer Applications

2300 Canyon Blvd

Boulder, CO 80302

WEATHERDATA, INC.

MIKE SMITH, PRESIDENT

\section{Certified Consulting Meteorologists}

"When Decisions Depend on Weather" • Custom Weather Forecasts \& Storm Warnings $\bullet$ Meteorological Investigations for Legal Matters, Expert Testimony $\bullet$ Weather \& Climate Data Analysis

245 N. Waco, Ste. 310

Wichita, KS 67202

316-265-9127 FAX: 316-265-0371

\section{METEOROLOGICAL APPLICATIONS}

JAMES H. MEYER, PRESIDENT

\section{Certified Consulting Meteorologist}

Forensic and Applied Meteorology • Climatology \& Environmental Research •Meteorological \& Air Quality Instrumentation \& Monitoring - Fugitive Dust \& Cooling Tower Drift Studies • Expert Testimony

\section{Allerton Lane}

Silver Spring, MD 20904

301-384-1875
COURTNEY CONSULTANTS, INC.

F.E. COURTNEY, PRESIDENT

GEORGE W. REYNOLDS, PH.D.

\section{Certified Consulting Meteorologists}

Meteorology, Modeling, Monitoring, Permits, Noise, Dust, Storms, Air Quality, Testimony, Accidents, Severe Weather

520 Carriage Dr

Atlanta, GA 30328

404-256-2487

AIR SCIENCE CONSULTANTS, INC.

SKYWATCHWEATHER CENTER ${ }^{\circledR}$

RICHARD J. MANCINI, PRESIDENT

\section{Certified Consulting Meteorologist}

Forecasting Services for Industry, Government \& News Media • Air Quality Modeling \& Monitoring $\bullet$ Stack Emissions Tests $\bullet$ Impact \& Climatological Studies • Forensic Meteorology

347 Prestley Rd.

Bridgeville, PA 15017

412-221-6000

WEATHER APPLICATIONS

DUANE R. (BOB) GOODMAN

\section{Certified Consulting Meteorologist}

Meteorology for Hazardous Waste Site Remediation, Climatology, Air Quality, Consulting, Forensic Meteorology

5812-314th St. Ct. S.

Roy, WA 98580

206-843-1374

METEOROLOGICAL STANDARDS

INSTITUTE

THOMASJ.LOCKHART

\section{Certified Consulting Meteorologist}

Quality Assurance Plans and Audits • System Specifications • Training Workshops in Measurement and Quality Assurance •Instrument Testing $\bullet$ Regulatory and Forensic Consulting

841 Seventh Lane, P.O. Box 26

Fox Island, WA 98333

206-549-2179

EINAR L. HOVIND

Certified Consulting Meteorologist

Industrial Meteorology • Air Quality Analysis • Weather Modification Services $\bullet$ Climatology $\bullet$ Forensic Meteorology

4152 Primavera $\mathrm{Rd}$

Santa Barbara, CA 93110

$805-964-8218$

Certified Consulting Meteorologist: The certification program of the American Meteorological Society is aimed at fostering the establishment and maintenance of a high level of professional competency, and mature and ethical counsel, in the field of consulting meteorology. Requirements of knowledge, experience, and character are determined by a five-person board. Objectives of the program and application procedures are described in full detail in the August 1993BULLETIN (pp. 1550-1554). 


\section{PROFESSIONAL DIRECTORY}

\section{SPACE RESERVED FOR CERTIFIED CONSULTING METEOROLOGISTS}

ALEXIS B. LONG, PH.D.

Certified Consulting Meteorologist

Weather Modification and Cloud Physics Research

70 Glenelg Drive

Mentone, Victoria 3194

Australia

(61)3-583-0621

METEOROLOGICAL EVALUATION

SERVICES, CO. INC. (MES)

PATRICK T. BRENNAN, PRESIDENT

JOHN R. MARTIN, VICE PRESIDENT

\section{Certified Consulting Meteorologists}

Air-Quality Consulting • Expert Testimony •Industrial Meteorology • Nuclear Licensing Studies • Weather Investigations for Legal and Insurance Firms

165 Broadway

Amityville, NY 11701

516-691-3395

\section{RAY HOXIT, PH.D.}

\section{Certified Consulting Meteorologist}

Detailed Weather Analyses - Forensic Meteorology and Expert Testimony • Climatological Studies $\bullet$ Specialized Weather Forecasts

P.O. Box 140

Lake Toxaway, NC 28747

704-884-7979

\section{STONE \& WEBSTER ENVIRONMENTAL} SERVICES

A. KASPRAK

C.A. MAZZOLA

Certified Consulting Meteorologists

Meteorological System Evaluations $\bullet$ Hazardous Chemical Modeling - New Source Review Permitting • Climatological Studies • Expert

Testimony • Air Quality/Nuclear Dispersion Modeling

P.O.Box 2325 Woodside Executive Office Park, Suite F-25 Boston, MA $02107 \quad 1359$ Silver Bluff Road, Aiken, SC 29803

617-589-2923 803-641-0420

Other offices in New York, NY; Cherry Hill, NJ; Houston, TX; Ft.

Lauderdale, FL; Tampa, FL; Denver, CO; Oak Ridge, TN.

\section{CLIMATOLOGICAL CONSULTING} CORPORATION

ROBERT G. FISHER

\section{Certified Consulting Meteorologist}

Specializing in Forensic Meteorology
6941 N. Camino de Fosforo

Tucson, AZ 85718-1011

602-797-2877
150 Shope Creek Road Asheville, NC 28805 704-298-4237

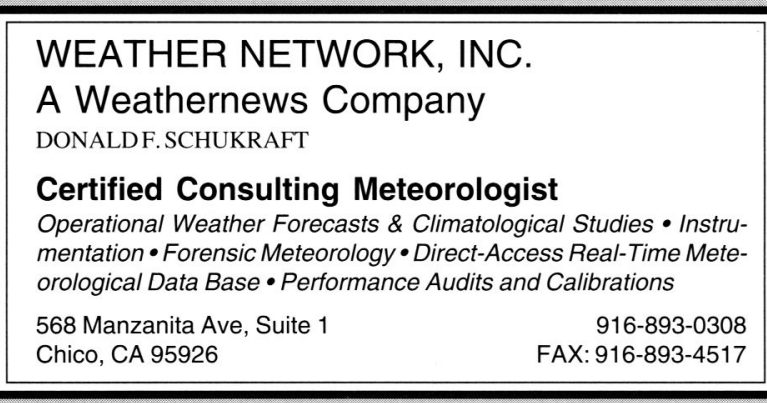

SIGMA RESEARCH CORPORATION

DR. STEVEN R. HANNA

DR. LLOYDL. SCHULMAN

JOSEPH S. SCIRE

Certified Consulting Meteorologists

Air Quality Modeling \& Development $\bullet$ Evaluation $\bullet$ Permitting $\bullet$ Data Analysis • Air Toxics $\bullet$ Acid Deposition • Ozone $\bullet$ Complex Terrain $\bullet$ Indoor Air Quality $\bullet$ Expert Testimony

196 Baker Avenue

Concord, MA 01742

FAX: 508-371-4280

\section{SPENCER DUCKWORTH}

\section{Certified Consulting Meteorologist}

Weather-Related Marine Incident Evaluations - USCG Licensed,

Vessel Operator $\bullet$ Registered Engineer in California

3403 Tanager Ave.

Davis, CA 95616

916-756-9558

WILLIAM H. KLEIN, PH.D.

\section{Certified Consulting Meteorologist}

Applied Research $\bullet$ Expert Testimony $\bullet$ Climate Studies $\bullet$ Extended Forecasting • Systems Design • Technique Development• Statistical Meteorology $\bullet$ Synoptic Climatology

5225 Pooks Hill Road \#929 South

$301-897-5485$

Bethesda, MD 20814
$508-371-4200$

JWR, INC.

JACK W. REED, PRESIDENT

\section{Certified Consulting Meteorologist}

Practice Limited to Problems with Airblast Propagations \& Hot-Air Ballooning

5301 Central NE, Suite 220

Albuquerque, New Mexico 87108

$505-265-6550$ 


\section{SPACE RESERVED FOR CERTIFIED CONSULTING METEOROLOGISTS}

\section{ENSR CONSULTING AND ENGINEERING \\ 20 OFFICES NATIONWIDE \\ BRUCEEGAN, Sc.D. \\ ROBERTIWANCHUK \\ Certified Consulting Meteorologists \\ Air Toxics Control • Regulatory Analysis - Acid Rain Programs • Permitting and Compliance $\bullet$ Model Research and Development $\bullet$ Air Quality Measurement $\bullet$ Engineering $\bullet$ Emergency Response Planning 35 Nagog Park \\ Acton, MA 01720 $800-722-2440$}

\section{WEATHERCOUNSEL}

FREDL.ZUCKERBERG

\section{Certified Consulting Meteorologist}

Consulting for Weather Sensitive Activities $\bullet$ Decision Strategies Algorithm Design $\bullet$ Training Needs Defined and Satisfied $\bullet$ Research Studies

2 Glenwood Lane

East Hills, NY 1157-1411

$516-621-7551$

\section{RADIAN CORPORATION}

RICHARD R. BOYD KARENKOWALEWSKY

HOWARDW. BALENTINE

\section{Certified Consulting Meteorologists}

Industrial Meteorology • Air Quality Modeling \& Monitoring • Remote Sensing • Environmental Impact • Risk Assessment • Emergency Response Planning $\bullet$ Hazardous Waste Management

Washington, DC

703-713-1500

Sacramento, CA 916-362-5332

\section{WEATHERVISION}

(Formerly Gulf Coast Weather Service)

ANDY JOHNSON, OFFICE MANAGER

\section{Certified Consulting Meteorologist}

Expert Testimony • Complete Computerized Data Gathering • Video Graphics • Digital Nowcasting $\bullet$ Industrial Forecasting

P.O. Box 31113

Tampa, FL 33631-3113

TWX: 810-876-0618 813-287-0900

FAX: 813-872-7202

\section{AWS SCIENTIFIC, INC.}

BRUCE H. BAILEY, PH.D., PRESIDENT

\section{Certified Consulting Meteorologist}

Atmospheric Field Research • Monitoring and Data Analysis • Applied Climatology • Instrumentation - Wind and Solar Energy • Engineering Meteorology $\bullet$ Modeling $\bullet$ Daylighting Simulation

3 Washington Square

Albany, NY 12205
518-869-5637 FAX: 518-869-5729

\section{ENSR CONSULTING AND ENGINEERING 20 OFFICES NATIONWIDE \\ DAVID HEINOLD DAVID SHEA \\ ROBERT PAINE \\ Certified Consulting Meteorologists \\ Air Toxics Control - Regulatory Analysis - Acid Rain Programs • Permitting and Compliance $\bullet$ Model Research and Development $\bullet$ Air Quality Measurement • Engineering • Emergency Response Planning 35 Nagog Park \\ Acton, MA 01720 $800-722-2440$}

\section{ALPINE GEOPHYSICS}

T.W. TESCHE, PH.D.

\section{Certified Consulting Meteorologist}

Urban Airshed Modeling $\bullet$ Meteorological and Air Quality Modeling and Analysis $\bullet$ Emission Inventory Development $\bullet$ Aerometric Field Program Design • Avalanche Forecasting

Post Office Box 2059

303-349-6118

Crested Butte, CO 81224 FAX 303-349-6229

ENSERCH ENVIRONMENTAL CORPORATION LAURENCE A. LABRIE DOUGLAS J. FULLE Certified Consulting Meteorologists

Air Quality Modeling $\bullet$ EnvironmentalMonitoring $\bullet$ Permitting $\bullet$ Expert Testimony $\bullet$ Environmental Software $\bullet$ Hazardous Waste Remediation Services • Environmental Consulting

Lyndhurst, NJ 201-460-1900; Denver, CO 303-988-2202 Atlanta, GA 404-449-5800; Boston, MA 617-451-1201 Bellevue, WA 206-451-4600; and other offices nationwide Formerly the Environmental Division of Ebasco Services Incorporation

DBS ASSOCIATES, INC

DAVID B. SPIEGLER, PRESIDENT

\section{Certified Consulting Meteorologist}

Weather, climate, and environmental products, services, and information systems $\bullet$ Applied research in the atmospheric sciences.

22 Fiske Road

Lexington, MA 02173
Lexington, MA: (617) 862-0888 Atlanta, GA: (404) 442-9298

\section{CLIMATOLOGICAL CONSULTING} CORPORATION

JEAN T. LEE

\section{Certified Consulting Meteorologist}

Specializing in Forensic Meteorology

1914 Peter Pan Street

Norman, OK 73072

405-321-8011
150 Shope Creek Road Asheville, NC 28805 704-298-4237

Certified Consulting Meteorologist: The certification program of the American Meteorological Society is aimed at fostering the establishment and maintenance of a high level of professional competency, and mature and ethical counsel, in the field of consulting meteorology. Requirements of knowledge, experience, and character are determined by a five-person board. Objectives of the program and application procedures are described in full detail in the August 1993BULLetin (pp. 1550-1554). 


\section{PROFESSIONAL DIRECTORY}

\section{SPACE RESERVED FOR CERTIFIED CONSULTING METEOROLOGISTS}

GALSON CORPORATION

R. LELAND DAVIS, PRESIDENT

Certified Consulting Meteorologist

Air Toxics Management $\bullet$ Dispersion Modeling $\bullet A I R-1 \odot$ and pcAIR-1(A)Air Toxics Management System $\bullet A Q /$ MeteorologicalMonitoring

- Source Testing • Environmental Permitting • Expert Testimony

6601 Kirkville Road

East Syracuse, NY 13057

315-432-0506

Offices in Rochester, NY; New York, NY; Philadelphia, PA; and

Berkeley, CA

\section{FORENSIC METEOROLOGY}

ASSOCIATES

WALTER A. LYONS, PH. D.

\section{Certified Consulting Meteorologist}

Expert Testimony and Consulting $\bullet$ Weather Reconstruction using Satellite and Radar $\bullet$ Winter and Severe Local Storms • Lightning $\bullet$ Air Quality • Climate Records • Video Time Lapse System Rentals

46050 Weld County Road 13

Ft. Collins, CO 80524

$800-854-7219$ FAX: 303-568-7664

TRINITY CONSULTANTS, INC.

D. BRUCE TURNER, SENIOR CONSULTANT

\section{Certified Consulting Meteorologist}

Air Quality Consulting Dispersion Modeling Courses Worldwide • BREEZE Dispersion Modeling PC Software • Expert Testimony

P.O. Box 2099

Voice and Fax: 919-967-0325

Chapel Hill, NC 27515-2099

Other Offices: Dallas; Kansas City; Atlanta;

Baton Rouge; Fort Collins, CO.

\section{WEATHER SUPPORT SERVICES}

BURTON L. SYLVERN, PRESIDENT

\section{Certified Consulting Meteorologist}

Specialist since 1955

Weather Investigations For Law Firms and Insurance Agencies• Expert Testimony $\bullet$ Climatological Studies

5690 Owls Nest Rd.

Tallahassee, FL 32308

904-668-0521

WEATHERBANK, INC.

STEVEN A. ROOT, CCM

PETER J. STOLL

MARK E. EUBANK JOSEPH R. NICHOLLS

\section{Certified Consulting Meteorologist}

Operational Forecasting • Broadcast Services $\bullet$ Satellite Transmission Network • Forensic Studies • Real TIme Meteorological Data Base • Climatological Research

5 Triad Center, Suite 315

Salt Lake City, UT 84180
ACCU-WEATHER INC.

ELLIOT ABRAMS, SENIOR VICE PRESIDENT

\section{Certified Consulting Meteorologist}

Meteorological consultants serving industry, government, and the media

619 W. College Avenue

State College, PA 1680

814-237-0309

\section{TASC}

ARTHUR BASS, PH.D.

\section{Certified Consulting Meteorologist}

Leadership in Applied Information Technologies • Applied Research Information Systems, Services, and Data Products for the Earth and Environmental Sciences

55 Walkers Brook Drive

$617-942-2000$

Reading, MA 01867

\section{ROBERT L. CARNAHAN}

\section{Certified Consulting Meteorologist}

Specializing in System Development, Meteorological User Requirement Studies and Environmental Public Contact Programs.

13 Lancaster Road

302-644-1809

Lewes, Delaware 19958 FAX: 302-644-0803

\section{JERRY A. WILLIAMS}

\section{Certified Consulting Meteorologist}

Specializing in Climate Studies • Mesoscale and Forensic Marine Meteorology $\bullet$ Expert Testimony

3220 Verdant Way

San Jose, CA 95117

408-249-4968 FAX:408-249-2239

\section{CLIMATOLOGICAL CONSULTING CORPORATION STEVEN BUSINGER, PH.D.}

\section{Certified Consulting Meteorologist}

Specializing in Forensic Meteorology

Department of Meteorology

University of Hawaii at Manoa

2525 Correa Road

Honolulu, Hawaii 96822

150 Shope Creek Road

(808) 956-8775

\section{Asheville, NC 28805-9795} (704) 298-4237 


\section{PROFESSIONAL DIRECTORY}

\section{SPACE RESERVED FOR CERTIFIED CONSULTING METEOROLOGISTS}

\section{PREDICTION AND EVALUATION SYSTEMS}

DR. ALLAN H. MURPHY

DR. EDWARD S. EPSTEIN

\section{Certified Consulting Meteorologists}

Applications of probability and statistics $\bullet$ Model/forecast verification - Design/evaluation of forecasting experiments • Use/value of weather/ climate information - Short courses

3115 NW McKinley Drive

Corvallis, OR 97330-1139

8216 Inverness Hollow Terrace Potomac, MD 20854-2627

503-754-7424 301-299-2259

\section{WAYNE R. SAND, PhD}

\section{Certified Consulting Meteorologist}

Specializing in Aviation Weather (Icing • Thunderstorms • Turbulence $\bullet$ Low Ceiling and Visibility $\bullet$ Windshear $\bullet$ Orographic Weather)

- Accident Weather Analysis • Expert Testimony

7020 Baseline $\mathrm{Rd}$.

Boulder, CO 80303-3141

\section{CLIMATOLOGICAL CONSULTING CORPORATION}

DOUGLAS A. STEWART, Ph. D.

\section{Certified Consulting Meteorologist}

Specializing in Forensic Meteorology

3071 Lucaya Street

Miami, FL 33133

305-856-4302

150 Shope Creek Road Asheville, NC 28805-9795 $704-298-4237$

\section{CLIMATOLOGICAL CONSULTING CORPORATION}

ROBERT N. SWANSON

\section{Certified Consulting Meteorologist}

Specializing in Forensic Meteorology

1216 Babel Lane

Concord, CA 94518

150 Shope Creek Road

(510) 676-2228

Asheville, NC 28805-9795

(704) 298-4237

\section{CLIMATOLOGICAL CONSULTING CORPORATION}

\section{LEE E. BRANSCOME, Ph. D.}

Certified Consulting Meteorologist

Specializing in Forensic Meteorology

7338 155th Place North

Palm Beach Gardens, FL 33418

150 Shope Creek Road

407-744-4889 Asheville, NC 28805-9795 704-298-4237

KIT K. WAGNER, Ph.D.

\section{Certified Consulting Meteorologist}

Meteorological Analysis \& Modeling • Air Quality Analysis \& Modeling

- Urban Airshed Modeling • Meteorological Research • Public Policy

- Meteorology \& Air Quality Training Programs

603 36th Ave. NW

Norman, OK 73072-4106

405-329-8707 FAX: 405-329-8717 


\section{PROFESSIONAL DIRECTORY}

\section{SPACE RESERVED FOR MEMBERS}

\author{
INTERNATIONAL WEATHER \\ CORPORATION \\ WALTER F.ZELTMANN \\ Forensic Meteorology • Applied Climatology \\ 719 Bay Ridge Ave. \\ Brooklyn, NY 11220
}

718-748-8066

WEATHERNEWS AMERICA INC.

ROBERT A. RAGUSO,

WALTER A. HACK, Senior Meteorologist Vice President, Marine ROBERT J. SPANIER, Meteorologist

\section{MARINE SERVICES}

Optimum Ship Routing $\bullet$ Weather Verification Surveys $\bullet$ Coastal \& Harbor Forecasts $\bullet$ Marine Weather Forecasts • Marine Operational Forecasts $\bullet$ Voyage Planning Studies

11 Commerce Drive

Cranford Business Park

$908-276-5456$

Cranford, NJ 07016

FAX: $908-276-5688$ TELEX: 6734296

\section{GEOMET TECHNOLOGIES, INC.}

MARK J. STUNDER

Environmental Expert Systems, Artificial Intelligence - Air Pollution Analysis, Modeling \& Monitoring • Environmental Data Systems, Climatological Studies $\bullet$ Research and Operations

20251 Century Blvd.

Germantown, MD 20874

$301-428-9898$

\section{CENTRAL WEATHER SERVICE}

EARL S. FINCKLE, PRESIDENT

\section{Consulting Meteorologists}

Radio Weathercasting Experts \& Commodity Weather Specialist • Aviation, Business \& Industry, Government, Forensic Meteorology • Over a Quarter of a Century of Experience

1098 S. Milwaukee Ave.

708-537-5920

Wheeling, IL 60090

FAX 708-537-5926

\section{C.W. THORNTHWAITE ASSOCIATES \\ WILLIAM J. SUPERIOR, PRESIDENT}

Research \& Field Studies - Sensors \& Systems - Publications in Climatology

Rte. 1, Centerton

Elmer, NJ 08318

$609-358-2350$

\section{WEATHER SURVEYS}

CONRAD B. GOSSET, M.S., FORENSIC METEOROLOGIST

Specializing in Investigations for Law Firms \& Insurance Companies

P.O. Box 67

Cross River, NY 10518

914-769-1350

WEATHERVISION

(formerly Gulf Coast Weather Service)

WTVT Television Weather Services

ROY LEEP, EXECUTIVE DIRECTOR

Complete Computerized Data Gathering • Video Graphics • Digital

Nowcasting •Industrial Forecasting $\bullet P C$ Remote Accessing

P.O Box 31113

Tampa, FL 33631-3113

TWX 810-876-0618

813-287-0900 FAX 813-872-7202

ACCU-WEATHER, INC.

JOEL N. MYERS, PH.D., PRESIDENT

ELLIOT ABRAMS, M.S., CCM, SENIOR VICE PRESIDENT

JOSEPH P. SOBEL, PH.D., SENIOR VICE PRESIDENT

Weather Forecasting $\bullet$ Real Time Meteorological Data Base $\bullet$ Satellite Radar, and Graphics Images • Climatological and Forensic Services

- Over 20 years of quality service

619 W. College Ave.

State College, PA 16801

814-237-0309

\section{KNIGHT-RIDDER/GLOBAL WEATHER} SERVICES

Crop Weather Impact Analysis via: Knight-Ridder Financial News • Knight-Ridder Financial Information • Commodity News Services • American Quotation Systems • UNICOM Inc.

P.O. Box 6053

Leawood, KS 66206

913-642-7373

Telex 42585

FLEETWEATHER, INC.

Since 1969

TORE H. JAKOBSEN, M.S., CO-OWNER

Forecasts for Weather-Sensitive Industry - Past Weather Research \& Expert Testimony • Global Ship-Guidance \& Surveillance $\cdot$ Weathercasting • Automated Weather Databases \& Delivery Systems

The Fleetweather Building, 1966 Route 52

Hopewell Jct., NY 12533

FAX 914-226-1918 914-226-8300 Telex 178994 


\section{PROFESSIONAL DIRECTORY}

\section{SPACE RESERVED FOR MEMBERS}

\section{BRUCE F. WATSON}

\section{Consulting Meteorologist}

Research Specializing in Weather \& Climate of Minnesota \& Bordering States \& Provinces for Application to Agriculture, Forensics, Engineering Studies, Environment, \& Marketing

2514 Brenner St.

Roseville, MN 55113

NASH C. ROBERTS, JR., CONSULTANTS, INC.

\section{Consulting Meteorologists Since 1946}

General Industrial • Marine • Forensic • Air Quality • Agricultural • Commodities $\bullet$ Television

1040 N. Rampart St.

New Orleans, LA 70176

504-581-1688

COMPU-WEATHER, INC.

\section{Forecast and Research Specialists}

Weather Forecasting for Media, Industry, Government • Research Studies • Consultation • Forensic Meteorology • Excellent Client References

29-50 Union St.

Flushing, NY 11354

$718-961-4242$

\section{YORK SERVICES CORPORATION}

\section{Environmental Science \& Air Permits}

Air Pollution Meteorology $\bullet$ Atmospheric Diffusion Modeling $\bullet$ Mete orological/Air Quality Monitoring Systems •Boundary-LayerProfiling

1 Research Drive

Stamford, CT 06906

Phone: 203-325-1371 Fax: 203-357-0166

\section{MATRIX MANAGEMENT GROUP}

\section{General Management Consultants}

Meteorological Consultants to Government \& Industry • Project Management • Organization • Mgmt. Audits • Mgmt. Training • Policy Studies • Bridging the Gap Between Meteorology and Public Works

466 Colman Bldg. Seattle, WA 98104

206-621-1977 206-842-4186
METEOTEC INC. - An Alaska Science \& Technology Co.

ROBERT L. BALDWIN, PRESIDENT

Environmental Science \& Engineering Consultants

Analysis $\bullet$ Systems $\bullet$ Sensing Communications $\bullet$ Automation $\bullet$ Wind \& Solar Energy • Air Quality • Noise • Modeling • Forensics • Management $\bullet$ Planning $\bullet R \& D$

P.O. Box 102915

907-562-2482

Anchorage, AK 99510

Telex 09026713 AKSCITECH AHG

BOYD E. QUATE \& ASSOCIATES

Specializing in Extended Period Weather Outlooks

P.O. Box 7065-Holland Station

Suffolk, VA 23437

$804-657-6531$

WEATHER CONSULTANTS OF CANADA CO. LTD (TELEWEATHER)

MORRIS KESTIN, PRESIDENT

Long-Range Weather Outlooks \& Detailed Forecasts for Marketing, Radio, TV, Movies, Publications, Commodities, Consultants to Indus-

try, Municipalities, Utilities, Forensic Meteorology since 1956

65 Marlborough Ave.

Toronto, Ont., Canada M5R 1X5

$416-968-7981$

LIGHTNING LOCATION \& PROTEC-

TION, INC.

Lightning Locating \& Warning Systems $\bullet$ Lightning \& Weather Radar Display Systems • Sensors for Automatic Meteorological Stations • Consulting on Lightning Hazards \& Lightning Protection

2705 E. Medina Rd.

Suite 111

Tucson, AZ 85706

$602-741-2838$

\section{WSI CORPORATION}

Serves customers who depend on high quality weather information • value-added data - software packages - workstations - markets include media, aviation, industry, and government

4 Federal St.

508-670-5000

Telex 95-1184

Billerica, MA 01821 


\section{PROFESSIONAL DIRECTORY}

\section{SPACE RESERVED FOR MEMBERS}

ESSCO - ENVIRONMENTAL SCIENCE AND SERVICES CORPORATION

WALTER G. HOYDYSH, PH.D., DIRECTOR

Wind Tunnel Modeling • GEP Stack Height Demonstration • Dispersion Modeling • Ambient Air Quality Monitoring • Pedestrian Level Wind Studies • Wind Pressure \& Aeroelastic Studies

45-43 37th St.

718-786-3948

Long Island City, NY 11101

FAX 718-706-0391

\section{FRED W. DECKER, PH.D.}

Meteorologist Since 1937 (USWB, AAF, USAFR, Universities, Forensic). Physics Expert

Attorneys' Expert for Weather Reconstructions - Testimony and Cross-Examination Advice - Professor • Forecaster • Author of The Weather Workbook, Weather Analysis, Weather for Lawyers

827 NW 31 st St.

Corvallis, OR 97330

$503-753-7271$

METRO WEATHER SERVICE, INC.

Servicing our clients for 15 years

Specialized Forecasts for the Media, Utilities \& Industry • Research

\& Consultation - TV Weathercaster Training

507-535 Rockway Ave.

Suite \#3

Valley Stream, NY 11581

$516-568-8844$

\section{TECHNI-WEATHER CENTER}

WILLIAM JACQUEMIN, CHIEF METEOROLOGIST

DOMINIC SCERBO, METEOROLOGIST

PROFESSIONAL SERVICES INCLUDING: Weather forecasting for Media, Utilities, Industry, Ski Areas, Government, Educational Svcs, Insurance/LawyerReports, Thunderstorm Research, Weatherphones
18 Woodside Ave

Danbury, CT 06810-7123
203-744-2064 FAX 203-790-9241

\section{ALLIED WEATHER CONSULTANTS}

JOSEPH ALLEN, CHIEF FORECASTER

ERWIN K. KAUPER, CCM

Weather Forecasting Services for Utilities, Agriculture, Construction, and the Film Industry

P.O. Box 4584

Covina, CA 91723

818-967-1098

\section{EARTH ENVIRONMENT SERVICE}

STEVE NEWMAN, CHIEF METEOROLOGIST

Agriculture • Site Forecasts $\bullet$ Media $\bullet$ Graphics $\bullet$ Expert Testimony • Worldwide Forecasting

8200 St. Helena Rd.

Santa Rosa, CA 95404

707-539-1603 FAX707-538-5441

\section{METEOROLOGICAL EVALUATION}

SERVICES, CO. INC. (MES)

MAYNARD E.SMITH FRANKP.CASTELLI MARKL. KRAMER

\section{Consulting Meteorologists Since 1968}

Air-Qualtiy Modeling and Analysis $\bullet$ Forensic Meteorology $\bullet$ Monitoring and Data Analysis • Nuclear and Industrial Permitting Studies

165 Broadway

Amityville, NY 11701

$516-691-3395$

SURFACE SYSTEMS, INC.

JOE R. KELLEY, PRESIDENT/CEO

RICHARD C. CURTIS, OPERATIONS MANAGER

DAVIDC. TRASK, CHIEF METEOROLOGIST

SPECIALIZING IN: Remote Roadway \& Runway Weather Information Systems • Pavement Specific Forecasting \& Meteorological Consulting for the Transportation Industry

11612 Lilburn Park Road

314-569-1002

St. Louis, MO 63146 FAX 314-569-3567

ROEMER WEATHER, INC. / WEATHER TRADES, INC.

JAMES ROEMER, PRESIDENT

Specializing in Commodity Trading \& hedging Strategies Based on World Weather Conditions $\bullet$ Managed Account Programs $\bullet$ Commodity Advisory Services • Trade Winds Newsletter

2411 Grand Ave., Des Moines, IA 50312

900 Ridgefield Dr., \#150, Raleigh, NC 27609

$515-282-0233$

GLOBAL WEATHER DYNAMICS, INC

Weather Services, Turnkey Systems

Worldwide Weather Services • Oceanographic Services • Forecasting -DataBanks • Applied Research • Surface and Satellite Communications $• D E C$-, $D C$-, and PC-hosted Weather Message Switches

2400 Garden Road Monterey, CA 93940

408-649-4500

TELEX 171803 GWDI MNTY FAX 408-649-3288 


\section{PROFESSIONAL DIRECTORY}

\section{SPACE RESERVED FOR MEMBERS}

WEATHERNEWS, INC.

HIROYOSHIISHIBASHI, PRESIDENT

dekita-Global Environmental Experts for:

Agriculture - Aviation - Broadcast Media $\bullet$ Construction • Energy • Forensic $\bullet$ Maritime $\bullet$ Offshore $\bullet$ Public Works

WEATHERNEWS AMERICA, INC

333 W. El Camino Real, \#270

408-522-8350

Sunnyvale, CA 94087

FAX 408-481-0667

\section{FOX WEATHER}

\section{ALANFOX, DIRECTOR}

Satellite Analyses - Remote Sensing Studies - Site Forecasts • Extended Outlooks
3924 W. Channel Islands Blvd.

Oxnard, CA 93035

$805-985-8743$

FAX 805-985-3706

\section{COMMERCIAL WEATHER SERVICES ASSOCIATION}

JEFFREY C. SMITH, EXECUTIVE DIRECTOR

The National trade association for the private weather industry • Brochure of professional weather forecasting and consulting companies and list of services is available by contacting CWSA.
655 Fifteenth St., NW, Suite 310

Washington, D.C. 20005
202-546-6993 FAX 202-546-2121
WINCHESTER AND ASSOCIATES, INC.

Consultants to Management

JAMES W. WINCHESTER, PRESIDENT

40 Years management experience-Weather Service and Marine Industries

Market Analyses, Program Evaluation \& Business Development • Guidance and Assistance on Proposals and Procurements • Preparation of Technical, Procedures \& Training Manuals

131 Fairway Drive

Pass Christian, MS 39571

601-452-3882

FAX: 601-466-3812

Branch Office P. O. Box 559

Orange Beach, AL 36561 205-981-9004

\section{AEROSPACE ENVIRONMENT}

ASSOCIATES (AEA)

PAUL JANOTA, PH.D., PRESIDENT

\section{Atmospheric Science and Technology}

Expertise in Applied Meteorology, Climatology, and Remote Sensing • Analyzing needs, developing business, and solving problems sensitive to the Aerospace Environment for Industrial and Government Clients

16 Hancock Street

Lexington, MA 02173

PHONE/FAX: 617-674-2981
ALDEN ELECTRONICS, INC.

Distribution of global weather information $\bullet$ Lightning data $\bullet N E X R A D$ - Satellite Communications Networks • Weather Information Data and Display

40 Washington Street

508-366-8851

Westboro, MA 01581

SCIENCE \& ENGINEERING ANALYSIS CORPORATION (SEACOR)

STEPHEN R. ANDERSEN

PETER P. MILLER

SIMS E. DUGGINS

DAVID J. PREY

Air Quality Permitting $\bullet$ Control Engineering $\bullet$ Meteorological and Air Quality Measurements • Air Quality Modeling • Emissions Measurements $\bullet$ Data Analysis $\bullet$ Boundary Layer Studies

4700 McMurray Dr., Suite 108

$303-226-4040$

Ft. Collins, CO 80525

\section{GALSON CORPORATION}

CHRISTOPHER BEDFORD. JOHNFERLITO,

RICHARD WALIKIS

Air Toxics Management $\bullet$ Dispersion Modeling $\bullet$ Mesoscale forecasting, climatological studies •pcAIR-1@Air Toxics Management System - AQ/Meteorological Monitoring - Source Testing - Environmental

Permitting $\bullet$ Expert Testimony

6601 Kirkville Road

East Syracuse, NY 13057

315-432-0506

Offices in Rochester, NY New York; Philadelphia, $P A$ and Berkeley, $C A$

\section{R. M. YOUNG COMPANY}

Meteorological Instruments since 1964

Sensors to Measure: Wind Speed $\cdot$ Wind Direction - Peak Gusts •

Temperature $\bullet$ Delta $T \cdot$ Dew Point $\bullet$ Relative Humidity $\bullet$ Precipitation

\section{GDS}

\section{The National Lightning Detection Network ${ }^{\mathrm{tm}}$}

Providing real-time and archive lightning data to a variety of users for energy management, damage assessment, and airline and public safety. Forensic lightning experts available.

$602-573-0090$

2705 E. Medina Road, Suite 111

Tucson, AZ 85706 


\section{PROFESSIONAL DIRECTORY}

\section{SPACE RESERVED FOR MEMBERS}

\section{METEO CONSULT}

HARRY A. OTTEN, MANAGING DIRECTOR

WIM D. VAN DEN BERG, PH. D., DIRECTOR OF RESEARCH

Worldwide Forecasting with emphasis on Europe, Media, Marine (legally recognized in Dutch law), Agriculture, Software, Research and Consulting, and Lightning Data

P.O. Box 617

6700 AP Wageningen

The Netherlands
GOODMAN'S METEOROLOGICAL

SERVICES, INC.

MICHAEL GOODMAN, METEOROLOGIST and PRESIDENT

Meteorological Instruments

Sensors to monitor: Temperature, wind, relative humidity, precipitation, barometric pressure, and lightning. Individual units, remote monitoring units, and weather stations.

275 Eve Street

Manchester, $\mathrm{NH}$ 03104-1558

TOLL FREE 800-497-0571 FAX: 603-669-1990

For professional card rates, please apply to:

Executive Director, American Meteorological Society, 45 Beacon Street, Boston, MA 02108-3693

\section{EMPLOYMENT ANNOUNCEMENTS}

The American Meteorological Society publishes a monthly newsletter announcing employment opportunities for positions in the atmospheric and oceanic fields.

Members and nonmembers may subscribe to the announcements at an annual (12 monthly issues) subscription rate of $\$ 15$ for AMS members and $\$ 30$ for nonmembers (Both members and nonmembers, kindly add $\$ 10$ for airmailing to a foreign address). After July 1, half-year subscriptions (for July through December issues) are available at one half of the above annual prices

Organizations with openings to be filled by qualified individuals seeking positions in the atmospheric and related fields should contact the American Meteorological Society, 45 Beacon Street, Boston, MA 02108-3693, 617-2272426, ext. 250 for announcement guidelines and charges. ASCII diskettes are invited. You may also submit announcements via internet: amsempl@aip.org. 